\title{
An Effective Power Dispatch Strategy for Clustered Micro-grids while Implementing Optimal Energy Management and Power Sharing Control using Power Line Communication
}

\author{
Ahmed M. A. Haidar ${ }^{\mathrm{ab}}$ \\ Senior Member, IEEE \\ ${ }^{a}$ Universiti Malaysia Sarawak \\ Samarahan, Sarawak, Malaysia \\ ${ }^{b}$ University of Southern Queensland \\ Toowoomba, Queensland, Australia
}

\author{
Adila Fakhar \\ Department of Electrical and \\ Electronics Engineering \\ Universiti Malaysia Sarawak \\ Samarahan, Sarawak, Malaysia
}

\author{
Kashem M. Muttaqi \\ Senior Member, IEEE \\ School of Electrical, Computer, and \\ Telecommunications Engineering \\ University of Wollongong \\ Wollongong, NSW, Australia
}

\begin{abstract}
The mitigation of uncertainty in the availability of power generation from micro-grids to enable renewable resources to be dispatched is a daunting task for the individual operators. Installing energy storage systems may reduce the impact of renewable energy intermittency. However, a peculiarity in energy management can be arisen, particularly, when different energy providers manage these resources. Hence, an intelligent utilization approach should be devised to maximize the benefits of using battery energy storage, since the cost of this system is the most expensive part. This paper proposes an effective power dispatch strategy for clustered micro-grids. The developed hybrid algorithm implements optimal energy management and power sharing control using binary data. Frequency shift keying (FSK) technique has been adopted for transmitting the binary signal over the power line communication (PLC). A part of the algorithm is utilized to deal with the optimal scheduling control while the other actuates the dynamic demand response based Photovoltaic (PV) power forecasting. The performance of the proposed approach has been validated using data collected from the practical network of "Bario, Sarawak". The presented results suggest that the implementation of the proposed strategy can improve the efficiency of the overall system, causing less operating cost and fast return.
\end{abstract}

Index Terms- Energy management, Clustered micro-grids, $P V$ power forecasting, Demand response, Battery storage.

\section{INTRODUCTION}

The economic growth of the rural areas mainly depends on sufficient renewable energy resources (RER) to accommodate the increasing demand for electricity. The use of these resources can speed up the development of smallscale industries, agriculture and domestic needs. However, managing the unpredictable nature of RER located in widespread areas and owned by different energy suppliers is a challenging problem. In fact, intelligent energy management in the existing systems did not receive yet any attention nor the current configurations were designed according to the structure of local communities [1].

Numerous energy management approaches are discussed in the literature. For instance, ref. [2] proposed a real-time home energy management scheme in which the schedulable appliances were connected with a smart meter. The authors applied the concept of a mixed integer program to formulate the optimization problem under various practical constraints. In [3], the authors proposed an approximate optimization algorithm for event-based demand response management, considering the appropriate voltage levels and network constraints in islanded micro-grids. Although the authors in [3] demonstrated the utilization of this approach, the management of controllable load for a large number of consumers and the micro-grid structure with its capacity were not clearly stated. Ref. [4] introduced a demand response scheme using a game theory algorithm. The loads were scheduled based on different tariffs. Here, the tariff has been calculated for all loads and hours to reschedule the peak hours. A real-time decision model was proposed in [5] for load management in industries, where a variable realtime pricing is deployed. The approach optimizes the future time pricing uncertainties and schedules the loads in future time slots along with the load management for current time slots after receiving the current pricing. A multi-objective algorithm for energy management in $\mathrm{AC} / \mathrm{DC}$ micro-grid was applied in [6] using advanced metering infrastructure. The authors utilized a new concept known as an operational value factor for the improvement of micro-grids efficiency. The studies in [7-11] focused on economic dispatch by optimally scheduling the renewable generation, energy storage and responsive load demand. Ref. [7] utilized an optimization algorithm based on a multi-period artificial bee colony for solving the economic dispatch problem. The approach in [7] considered analyzing the performance of consumers in responsive load demand characteristics. Additionally, the combination of a bee colony and Markov chain algorithms was employed for predicting the uncertainties of generation and load demand. Despite the verification of the energy management system in [7] using real-time data, details about the capacity of renewable generators and the binary demand response control signal were not clearly reported. The authors in [8] applied the artificial bee colony for demand response management of micro-grid with the quasi-static algorithm. Likewise, the risk management scheme was implemented in [9] during the unscheduled islanding of grid-connected micro-grid. An energy management approach using multi-agent systems was proposed in [10] for a small hydro and PV generation systems at high altitude. In a multi-agent environment, the renewable distributed resources are controlled based on the local information via the energy management system. However, the study was limited in the availability of a communication mechanism for transferring the information between multi-agents. The multi-agent system was also used in [11] by employing a consensus algorithm for optimal resource management. The proposed dynamic energy management system in [12], utilized the information decision theory as a technique to handle the uncertainties of micro-grid. For the optimal operation of a micro-grid, ref. [13] introduced an intelligent dynamic energy management system capable of maintaining the supply to critical loads. In [14], the authors proposed agent-based modelling for the energy management of micro-grid. This study focused on solving the optimization problem of individual objectives 